\title{
Effect of pentoxifylline on colon cancer patients treated with chemotherapy (Part I)
}

\author{
Amichay Meirovitz ${ }^{\mathrm{a}}$, Lea Baider ${ }^{\mathrm{a}}$, Tamar Peretz ${ }^{\mathrm{a}}$, Samir Stephanos ${ }^{\mathrm{b}}$ and Vivian Barak ${ }^{\mathrm{a}, *}$ \\ ${ }^{a}$ Department of Oncology, Hadassah Medical Center and Faculty of Medicine, \\ Hebrew University of Jerusalem, Jerusalem, Israel \\ ${ }^{\mathrm{b}}$ Psychosomatic Department, Ulm University Medical Center, Ulm, Germany
}

Received 6 July 2021

Accepted 22 November 2021

\begin{abstract}
.
BACKGROUND: Cancer progression is associated with significant systemic clinical manifestations including cachexia induced weight loss and anorexia. Pentoxifylline (PTX) is a drug that has been shown to have multiple beneficial effects in cancer patients through its anti-inflammatory properties.

MAIN OBJECTIVE: To evaluate PTX effects on colon cancer patients treated with chemotherapy.

PATIENTS and METHODS: Forty metastatic colon cancer patients receiving chemotherapy were enrolled in this randomized study. 17 patients were treated with a full dose of PTX (400 mg TID), 9 patients with a reduced dose PTX (200 mg TID) and 23 served as controls (no PTX).

RESULTS: Follow-up evaluations of patients included the following: physical examination; leukopenia determination; weight determination; stomatitis determination; and survival rate. Patients treated with PTX (both full and reduced doses), experienced a significant increase in weight and a reduction in stomatitis relative to the control group. Treatment with PTX also significantly increased patient survival rate. All patients treated with PTX, had a median overall survival (OS) rate of 20.4 months as compared to 13.2 months in the control group.

CONCLUSIONS: PTX treatment of colon cancer patients, in addition to chemotherapy, significantly improved survival rates, induced weight gain and reduced stomatitis occurrence - all important parameters of cachexia.
\end{abstract}

Keywords: Pentoxifylline, colon cancer, cachexia, weight gain, stomatitis, survival

\section{Introduction}

Cancer progression is associated with significant systemic clinical manifestations including cachexia-induced weight loss and anorexia. Cachexia is a complex metabolic syndrome associated with multiple chronic or end-stage diseases and is characterized by loss of muscle mass with or without an accompanying loss of fat mass. While various treatment options are recommended for cancer-related cachexia, a number have proven to be ineffective, including cyproheptadine, hydrazine and metoclopramide, with progestogens being the only treatment currently approved in Europe [1].

Pentoxifylline (PTX), a xanthine derivative, has been shown to have a marked effect on cellular mediators of inflammation and tissue injury. Cancer cachexia has been linked to elevated levels of

\footnotetext{
*Corresponding author: Prof. Vivian Barak, Head, Immunology Laboratory for Tumor Diagnosis, Department of Oncology, Hadassah-Hebrew University Medical Center, Ein Kerem, P.O.B.12000, Jerusalem 9112001, Israel. Tel.: +972 0522401916; E-mails: barakvivi@hadassah.org.il; barakvivi@gmail.com
} 
inflammatory cytokines, including Tumor Necrosis Factor-alpha (TNF $\alpha$ ), IL-6 and IL-8. PTX has been shown to inhibit TNF $\alpha$ production, possibly via inhibition of TNF $\alpha$ and IL-1 mRNA transcription with relative preservation or even an increase of IL-10, an anti-inflammatory cytokine [2, 3]. In a separate study, PTX was shown to decrease TLR-mediated TNF $\alpha$ mRNA while increasing IL-10 mRNA $[4,5]$.

It has also been suggested that PTX reduces chemotherapy-induced stomatitis through the effect of these same inflammatory cytokines. In one study, a combination of PTX and vitamin E was assessed for the treatment of radiotherapy-induced oral mucositis. The combination decreased the duration of oral mucositis and dysphagia, and the occurrence of dysgeusia and fatigue without compromising locoregional control. This study concluded that the combination of PTX and vitamin E reduced the severity and duration of acute radiotherapy-induced oral mucositis and dysphagia in HNC patients [6, 7].

PTX not only possesses anticancer activity itself but increases cancer cell susceptibility to radiation therapy while reducing resultant long-term side effects $[1,2]$. Recently suggested treatments for cancer-related cachexia have involved different therapeutic combinations including PTX, anti-TNF $\alpha$ monoclonal antibodies (MoAb) and selective COX-2 inhibitors. Neonatal plasma factors were shown to contribute to the anti-inflammatory effects of PTX in new-born blood that were independent of soluble TNF $\alpha$ Receptor levels, p38 MAPK phosphorylation and $\mathrm{I} \kappa \mathrm{B}$ degradation [2, 8].

One of the latest approaches in anti-cancer therapeutics includes utilizing PTX in combined anticancer therapies [9-12]. A double blind, randomized, placebo-controlled clinical trial, demonstrated that PTX did not have any significant effect on weight gain in cachectic patients, but improved shortterm quality of life [11]. This is an exception, as majority of papers on the subject show positive results of PTX on weight gain and stomatitis.

The main aim of this study is to evaluate the effects of PTX on cachexia-related end points, including weight gain, stomatitis occurrence, blood counts, and on overall survival rate.

\section{Patients and methods}

Forty patients diagnosed with metastatic colon cancer were enrolled in this randomized study.

All patients were receiving chemotherapy, a combination of 5-fluorouracil (5-FU) and leucovorin, administered for 5 consecutive days, every 3 weeks until progression of disease or death.

Patients were randomized to either the treatment arm, receiving PTX or the control arm- no PTX.

A total of 17 patients were placed into the treatment arm, where 8 patients received a full PTX dose (400 mg, TID) and 9 patients received a reduced PTX dose $(200 \mathrm{mg}$, TID). These 9 patients did not tolerate the PTX treatment well due to side effects (including nausea, headache or dizziness) and as a result received treatment for a few weeks only. A total of 23 patients were randomized as controls and did not receive any PTX.

The study was reviewed, conducted and approved by the Hadassah - Hebrew University Medical Center Helsinki Committee (0346-12-HMO, 1.1.17).

All patients were assessed every 3 weeks (First 3 weeks - Time point 1, 6 weeks - Time point 2, 9 weeks - Time point 3 based on the following criteria and measurements:

* Physical examination

* Whole blood counts

* Weight measurement

* Stomatitis development

* Overall survival (OS calculated from initiation of chemotherapy with PTX). 


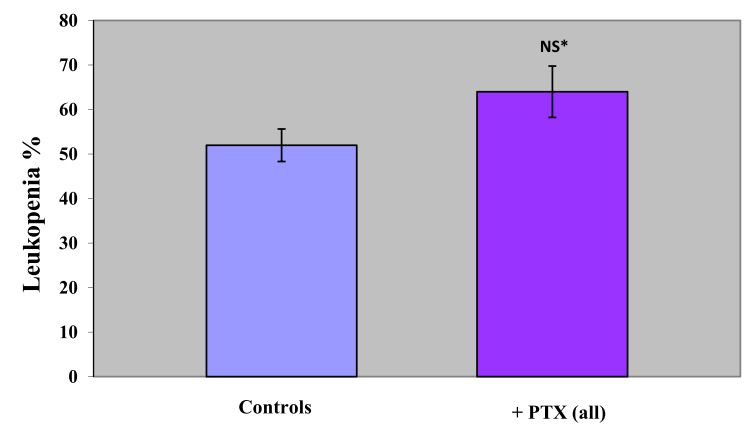

*NS = Not Significant

Fig. 1. Leukopenia of PTX-treated and control patients.

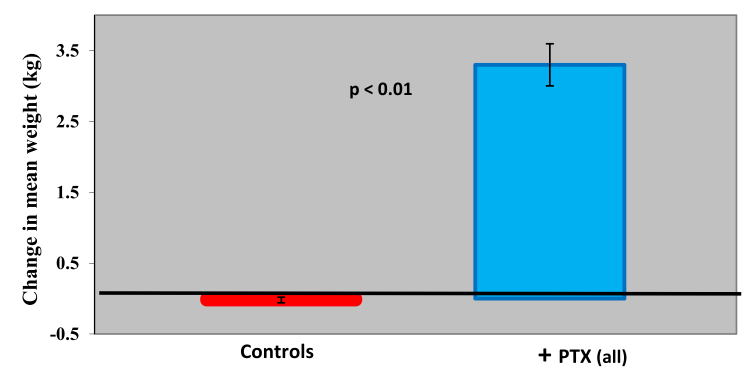

Fig. 2. Change in mean weight of groups in PTX-treated and control patients.

\subsection{Statistical analysis}

All statistical tests were performed using the SPSS statistical package.

\section{Results}

The results of the study include the following end point measurements:

- Whole blood counts - Leukopenia

No statistically significant difference was found between the PTX-treated patients and the control group (see Fig. 1). 5-FU is notorious for affecting bone marrow and platelet blood counts (all lines), however compared to other chemotherapies the effect is relatively modest. Since PTX has no major bone marrow effects bone marrow was not affected in the patients of this study.

- Weight - patients treated with PTX (both the full and reduced doses), gained a significant amount of weight during the treatment period as compared to the controls. The mean increase in weight of PTX-treated patients was $3.3 \mathrm{~kg}$, where a significant mean weight reduction of $0.2 \mathrm{~kg}$ in the control group was seen (Fig. 2) $(p<0.01)$.

- Stomatitis - the presence of stomatitis was assessed in patients treated with PTX (both full and reduced doses) and in the controls group. A gradual decrease in stomatitis cases was observed when comparing controls vs PTX full dose or PTX reduced dose: $45.5 \%$ of the control patients 


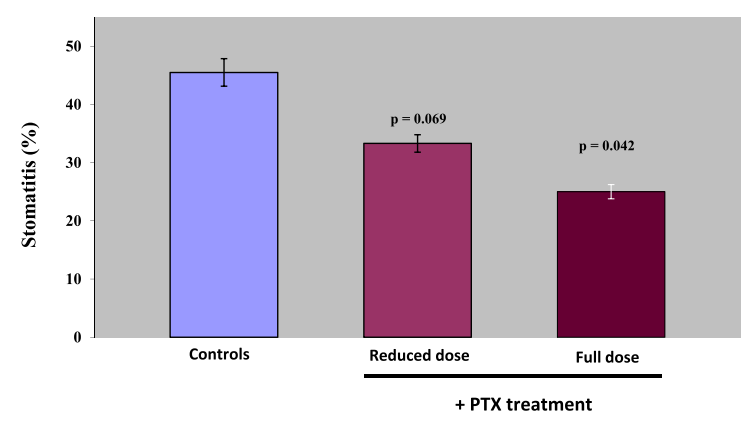

Fig. 3. Stomatitis (\%) in PTX-treated and control patients.

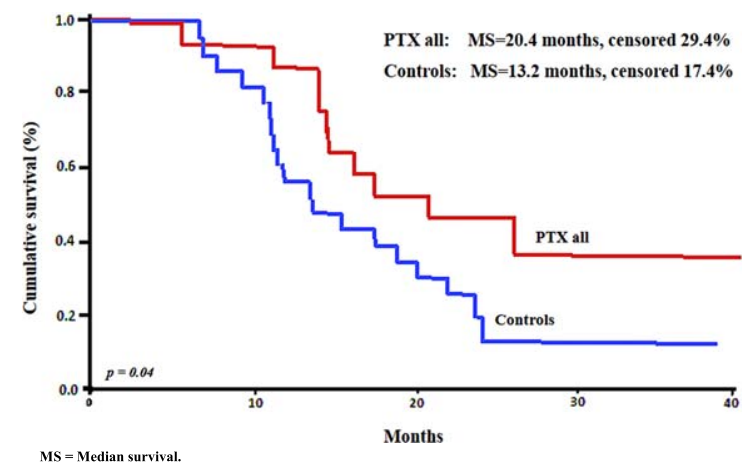

Fig. 4a. Cumulative survival of PTX-treated patients and controls.

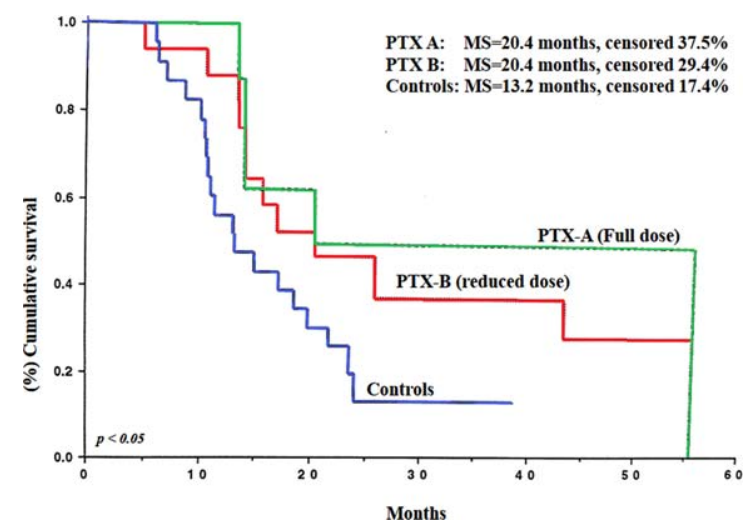

Fig. 4b. Cumulative survival of PTX-treated and control patients.

developed stomatitis, $33.3 \%$ of PTX reduced dose patients developed stomatitis, while only $25 \%$ of full dose PTX patients developed stomatitis (Fig. 3) $(p<0.05)$.

- Survival - treatment with PTX had a beneficial effect on survival. Patients treated with the full dose of PTX (400 mg, TID) had a median survival time of 20.4 months and $37.5 \%$ of them censored.

Patients treated with the reduced PTX dose (PTX $200 \mathrm{mg}$, TID) also had a median survival time of 20.4 months, but $29.4 \%$ of them censored. The control group had a median survival time of 13.2 
months and only $17.4 \%$ of patients censored $(p<0.05)$ (Fig. 4a). Survival follow up of patients was performed up to 5 years post-study initiation, providing significant insight into long-term effects (Fig. 4b).

The initiation of the increasing effects of PTX on survival can clearly be seen from the Kaplan Meyer graphs 8 months post-study initiation (Fig. 4a).

Patient demographics and specifics are summarized in the following Tables 1-4:

Table 1

Characteristics of PTX-treated cancer patients and controls

\begin{tabular}{|c|c|c|c|c|}
\hline Patient No. & Age $(y)$ & Sex & Height $(\mathrm{cm})$ & Sites \\
\hline \multicolumn{5}{|l|}{ PTX treated } \\
\hline 11 & 61 & $\mathrm{M}$ & 175 & Recto-sigmoid \\
\hline 12 & 72 & $\mathrm{M}$ & 174 & Colon \\
\hline 14 & 63 & $\mathrm{~F}$ & 177 & Recto-sigmoid \\
\hline 2 & 53 & $\mathrm{~F}$ & 184 & Colon \\
\hline 28 & 58 & $\mathrm{M}$ & 180 & Colon \\
\hline 6 & 48 & $\mathrm{M}$ & 169 & Colon \\
\hline 7 & 48 & $\mathrm{M}$ & 163 & Recto-sigmoid \\
\hline 8 & 69 & $\mathrm{M}$ & 155 & Recto-sigmoid \\
\hline 15 & 58 & $\mathrm{M}$ & 166 & Colon \\
\hline 17 & 67 & $\mathrm{M}$ & 153 & Recto-sigmoid \\
\hline 19 & 72 & $\mathrm{~F}$ & 175 & Colon \\
\hline 20 & 70 & $\mathrm{M}$ & 168 & Colon \\
\hline 24 & 68 & $\mathrm{~F}$ & 162 & Colon \\
\hline 27 & 52 & $\mathrm{~F}$ & 162 & Colon \\
\hline 3 & 71 & $\mathrm{M}$ & 157 & Recto-sigmoid \\
\hline 4 & 75 & $\mathrm{~F}$ & 178 & Recto-sigmoid \\
\hline 9 & 64 & $\mathrm{~F}$ & 150 & Colon \\
\hline \multicolumn{5}{|l|}{ Controls } \\
\hline 1 & 22 & $\mathrm{M}$ & 174 & Recto-sigmoid \\
\hline 10 & 64 & $\mathrm{~F}$ & 165 & Colon \\
\hline 13 & 42 & $\mathrm{M}$ & 160 & Recto-sigmoid \\
\hline 16 & 41 & $\mathrm{~F}$ & 160 & Recto-sigmoid \\
\hline 18 & 76 & $\mathrm{M}$ & 161 & Recto-sigmoid \\
\hline 21 & 67 & $\mathrm{~F}$ & 161 & Recto-sigmoid \\
\hline 22 & 70 & $\mathrm{M}$ & 165 & Colon \\
\hline 23 & 68 & $\mathrm{~F}$ & 163 & Colon \\
\hline 25 & 41 & $\mathrm{~F}$ & 179 & Colon \\
\hline 26 & 50 & $\mathrm{M}$ & 158 & Recto-sigmoid \\
\hline 29 & 65 & $\mathrm{~F}$ & 145 & Colon \\
\hline 30 & 72 & $\mathrm{~F}$ & 165 & Recto-sigmoid \\
\hline 31 & 59 & $\mathrm{M}$ & 173 & Recto-sigmoid \\
\hline 32 & 64 & $\mathrm{M}$ & 166 & Recto-sigmoid \\
\hline 33 & 71 & $\mathrm{M}$ & 169 & Colon \\
\hline 34 & 49 & $\mathrm{M}$ & 178 & Colon \\
\hline 35 & 42 & $\mathrm{M}$ & 176 & Recto-sigmoid \\
\hline 36 & 69 & $\mathrm{~F}$ & 161 & Recto-sigmoid \\
\hline 37 & 55 & $\mathrm{M}$ & 173 & Colon \\
\hline 38 & 54 & $\mathrm{M}$ & 174 & Colon \\
\hline 39 & 68 & $\mathrm{~F}$ & 145 & Recto-sigmoid \\
\hline 40 & 59 & $\mathrm{~F}$ & 156 & Recto-sigmoid \\
\hline 5 & 65 & $\mathrm{M}$ & 160 & Colon \\
\hline
\end{tabular}


Table 2

Colon Cancer patients - Concomitant diseases and medications

\begin{tabular}{|c|c|c|}
\hline Patient No. & Diseases* & Treatments** \\
\hline \multicolumn{3}{|l|}{ Controls } \\
\hline 1 & 1,3 & Convertin, Diabinese \\
\hline 10 & 4 & Tegretol, Idantoin, Mysoline \\
\hline 13 & 5 & - \\
\hline 16 & 5 & - \\
\hline 18 & 1 & Daonil \\
\hline 21 & 2,4 & Normiten, Cardoxin, Thiazide \\
\hline 22 & 1,4 & Normiten, Pressolat \\
\hline 23 & 4 & Nopan, Clonex \\
\hline 25 & 4 & - \\
\hline 26 & 4 & - \\
\hline 29 & 3,4 & Daonil, Cimetidine, Zantac \\
\hline 30 & 4 & Eltroxin, Idantoin, Dexamethasone, Zantac \\
\hline 31 & 4 & Pancreatin, Metamucil \\
\hline 32 & 5 & - \\
\hline 33 & $1,2,3,4$ & Normiten, Glucophage, Daonil. \\
\hline 34 & 5 & - \\
\hline 35 & 5 & - \\
\hline 36 & 5 & - \\
\hline 37 & 4 & Tagamet \\
\hline 38 & 1,3 & Normiten, Glibetic \\
\hline 39 & 2 & Aspirin \\
\hline 40 & 4 & Digoxin, Aspirin \\
\hline 5 & 5 & - \\
\hline \multicolumn{3}{|l|}{ PTX treated } \\
\hline 11 & $1,2,4$ & Nitroglycerin, Percocet \\
\hline 12 & 5 & - \\
\hline 14 & 5 & - \\
\hline 2 & $1,2,4$ & $\begin{array}{l}\text { Digoxin, Fusid, SlowK, Ikacor, Losec, Clexane, Cordil; Tiloptic, } \\
\text { Pilocarpine, Normiten, Pressolat, Zylol }\end{array}$ \\
\hline 28 & 4 & Clexane \\
\hline 6 & 4 & - \\
\hline 7 & 5 & - \\
\hline 8 & 5 & - \\
\hline 15 & 4 & Tagamet, +MMC, Zn \\
\hline 17 & 4 & Sucralfate, Zantac, Percocet \\
\hline 19 & 5 & HRT \\
\hline 20 & 2,4 & Adalat, Cordil, Lovalip, Convertin, Aspirin, Percocet \\
\hline 24 & 5 & \\
\hline 27 & $1,2,4$ & Lopressor, Atarax \\
\hline 3 & $1,2,4$ & Ikacor, Cordil, Cardoxin, Zantac, Normiten. \\
\hline 4 & 4 & Ferrocal \\
\hline 9 & $1,3,4$ & Convertin, Normiten, Dilatin \\
\hline
\end{tabular}

Diseases*: 1- Diabetes Mellitus, 2- IHD, 3-Hypertension, 4-other, 5-None. 
Table 3

Comparison between PTX-treated and control patients

\begin{tabular}{lccc}
\hline & PTX-treated & Controls & Significance \\
\hline Sex - M/F & $10 / 7$ & $13 / 10$ & NS \\
Age - mean (y) & 62.9 & 58.8 & NS \\
Height - females (cm) & 159 & 160 & NS \\
Height - males (cm) & 168.2 & 171.8 & NS \\
Height - all $(\mathrm{cm})$ & 166.9 & 164.2 & NS \\
Diagnosis: & & & \\
$\quad$ Colon cancer & 10 & 10 & NS \\
Recto-sigmoid & 7 & 13 & NS \\
Stage & Metastatic & Metastatic & NS \\
\hline
\end{tabular}

Table 4

Side effects of all study patients

\begin{tabular}{lcc}
\hline & PTX treated $(n=17)$ & Controls $(n=23)$ \\
\hline Leukopenia & 11 & 12 \\
Thrombocytopenia & 7 & 9 \\
Vomiting & 6 & 4 \\
Nausea & 8 & 6 \\
Diarrhea & 7 & 7 \\
Stomatitis & 5 & 10 \\
Alopecia & 1 & 1 \\
\hline
\end{tabular}

\section{Discussion}

This study showed that both reduced and full dose PTX in metastatic colon cancer patients receiving a combination of 5-FU and leucovorin had beneficial effects in terms of survival, cachexia and select side effects of chemotherapy (stomatitis and weight loss). These effects were demonstrated for all patients receiving PTX, both in full and reduced treatment doses.

PTX has been shown as a potent and efficacious inhibitor of TLR-mediated inflammatory cytokines in new-born cord blood and a promising neonatal anti-inflammatory agent [2]. All patients treated with PTX had a median OS of 20.4 months as compared to only 13.2 months in the control group. Moreover, $37.5 \%$ of patients receiving the full dose of PTX and 29.4\% receiving the reduced dose were alive about 5 years post-initiation of the study as compared to $17.4 \%$ in the control group. This significant result incurs interest and shows potential for future studies assessing the effects of low dose PTX treatment in cancer patients. In addition, PTX may be assessed with different combinations and regimens of multi-drug chemotherapies. Even if life expectancies do not change significantly with these approaches, quality of life of patients may be improved.

In PTX-treated patients, a significant weight gain was observed (mean $3.2 \mathrm{~kg}$ ) as compared to the control group, wherein an opposing effect, weight reduction occurred (mean $0.2 \mathrm{~kg}$ ). Weight is not only considered the optimal clinical parameter for cancer-related cachexia progression but is also strongly associated with overall patient well-being $[11,13,14]$.

Stomatitis is a major side effect of 5-FU based chemotherapy, causing significant morbidity. Patients treated with PTX developed stomatitis to a lesser degree than those in the control group. This fact 
together with the weight gain observed in the PTX-treated patients is an evidence of improved quality of life in such patients.

Results of one study showed that PTX in the treatment of cancer cachexia did not have any effect on weight gain in cachectic patients, however with short-term (1 month) treatment the quality of life was improved [11]. In other studies, PTX reduced the side effects of various treatments [12, 13].

The results of this study were comparable to those of other controlled clinical studies $[6,7,11,13$, 14]. While our study included only 40 patients, the other studies were performed on 70 patients [11], 59 patients [6] and 60 patients [7]. In our study part II [15], we will show the beneficial effects of PTX caused by decreasing levels of inflammatory cytokines $v s$ their increases in the controls.

In acute inflammatory diseases such as sepsis or current pandemics, such as COVID-19, patients are displaying a severe inflammatory cytokine syndrome referred to as a "cytokine storm".

Recently, it has been shown that COVID-19 causes severe lung inflammation, often leading to death. Preliminary results on using PTX in the COVID-19 pandemic for treatment of the cytokine storm, which is characteristic of patients suffering from a severe state of the disease, were published this year $[16,17]$.

High inflammatory cytokines, have been documented in patients with coronary artery disease [18], and PTX has been shown to reduce this pro-inflammatory process [19-21].

With regards to survival time, we evaluated patients for about 5 years post-study initiation, while other studies had a more limited follow up time of 6 months and 2 years [6,7]. The parameters assessed in the majority of studies were blood tests, weight assessment, stomatitis and OS, as in our study.

\section{Conclusion}

This randomized study demonstrated that PTX diminishes cachexia-related parameters, including weight gain, stomatitis and most importantly improves overall survival, in colon cancer patients treated with chemotherapy.

PTX, which is known to reduce the production of inflammatory cytokines and tumor markers (which will be demonstrated in Part II of this study [15], shows significant potential as a beneficial drug in severe symptomatic colon cancer patients.

\section{Acknowledgments}

We are thankful to: Dr. Rentschler Nicolaus for the PTX support, Dr. Kim Sheva for editing help, and Mrs. Shani Cohen for technical help.

\section{Funding}

The authors report no funding.

\section{Author contributions}

DATA CURATION: AM and TP

ANALYSIS OF DATA: LB, SS and VB

PREPARATION OF THE MANUSCRIPT: VB

REVISION FOR IMPORTANT INTELLECTUAL CONTENT: VB 


\section{References}

[1] Tazi E, Errihani H. Treatment of cachexia in oncology. Indian J Palliat Care. 2010; 16(3):129-37. doi: 10.4103/09731075.73644.

[2] Speer EM, Dowling DJ, Ozog LS, Xu J, Yang J, Kennady G, et al. Pentoxifylline inhibits TLR- and inflammasomemediated in vitro inflammatory cytokine production in human blood with greater efficacy and potency in newborns. Pediatr Res. 2017; 81(5):806-816. doi: 10.1038/pr.2017.6.

[3] Green LA, Kim C, Gupta SK, Rajashekhar G, Rehman J, Clauss M. Pentoxifylline reduces tumor necrosis factor- $\alpha$ and HIV-induced vascular endothelial activation. AIDS Res Hum Retroviruses. 2012; 28 (10):1207-15. doi: 10.1089/ AID.2011.0385.

[4] González-Espinoza L, Rojas-Campos E, Medina-Pérez M, Peña-Quintero P, Gómez-Navarro B, Cueto-Manzano AM. Pentoxifylline decreases serum levels of $\mathrm{TNF} \alpha$, Interleukin 6 and C-reactive protein in hemodialysis patients: results of a randomized double-blind, Controlled clinical trial. Nephrol Dial Transplant. 2012; 27(5):2023-8. doi: 10.1093/ndt/gfr579.

[5] Barancik M, Bohacova V, Gibalova L, Sedlak J, Sulova Z, Breier A. Potentiation of anticancer drugs: effects of Pentoxifylline on neoplastic cells. Int J Mol Sci. 2012; 13(1):369-82. doi: 10.3390/ijms13010369.

[6] Delanian SE, Lenglet T, Maisonobe T, Resche-Rigon M, Pradat PF. Randomized, Placebo-Controlled Clinical Trial Combining Pentoxifylline-Tocopherol and Clodronate in the Treatment of Radiation-Induced Plexopathy. Int J Radiat Oncol Biol Phys. 2020; 107(1):154-162. doi: 10.1016/j.ijrobp.2020.01.002. Epub 2020 Jan 24. PMID: 31987975.

[7] Sayed R, El Wakeel L, Saad AS, Kelany M, El-Hamamsy M. Pentoxifylline and vitamin E reduce the severity of radiotherapy-induced oral mucositis and dysphagia in Head and Neck Cancer patients: a randomized, Controlled study. Med Oncol. 2019; 37(1):8. doi: 10.1007/s12032-019-1334-5.

[8] Golunski G, Woziwodzka A, Piosik J. Potential Use of Pentoxifylline in Cancer Therapy. Curr Pharm Biotechnol. 2018; 19(3):206-216. doi: 10.2174/1389201019666180528084641.

[9] Napolitano R, De Matteis S, Lucchesi A, Carloni S, Cangini D, Musuraca G, et al. Pentoxifylline-Induced Apoptosis in Chronic Lymphocytic Leukemia: New Insights into Molecular Mechanism. Mini Rev Med Chem. 2018; 18(3):287-294. doi: 10.2174/1389557517666171002162258.

[10] Owosho AA, Estilo CL, Huryn JM, Yom SK. Pentoxifylline and tocopherol in the management of cancer patients with medication-related osteonecrosis of the jaw: an observational retrospective study of initial case series. Oral Surg Oral Med Oral Pathol Oral Radiol. 2016; 122(4):455-9. doi: 10.1016/j.oooo.2016.06.019.

[11] Mehrzad V, Afshar R, Akbari M. Pentoxifylline treatment in patients with cancer cachexia: A double-blind, randomized, placebo-Controlled clinical trial. Adv Biomed Res. 2016; 22;5:60. doi: 10.4103/2277-9175.179182.

[12] Price ML, Lai YE, Marcus KL, Robertson JB, Lascelles BDX, Nolan MW. Early radiation-induced oral pain signaling responses are reduced with pentoxifylline treatment. Vet Radiol Ultrasound. 2021; 62(2):255-263. doi: 10.1111/ vru. 12943.

[13] Tisdale MJ. Cachexia in Cancer patients. Nat Rev Cancer. 2002; 2:862-71. doi: 10.1038/nrc927.

[14] Persson C, Glimelius B. The relevance of weight loss for survival and quality of life in patients with advanced gastrointestinal cancer treated with palliative chemotherapy. Anticancer Res. 2002; 22:3661-8.

[15] Meirovitz A, Baider L, Peretz T, Stephanos S and Barak V. PTX treatment of Colon Cancer (Part II) - Mode of action and effects on Tumor Markers and Cytokines. (to be published shortly, 2021).

[16] López-Iranzo FJ, López-Rodas AM, Franco L, López-Rodas G. Pentoxifylline and Oxypurinol. Potential Drugs to Prevent the "Cytokine Release (Storm) Syndrome" Caused by SARS-CoV-2. Curr Pharm Des. 2020; 26(35):4515-4521. doi: 10.2174/1381612826666200811180232.

[17] González-Pacheco H, Amezcua-Guerra LM, Sandoval J, Arias-Mendoza A. Potential usefulness of Pentoxifylline, a non-specific phosphodiesterase inhibitor with anti-inflammatory, anti-thrombotic, antioxidant, and anti-fibrogenic properties, in the treatment of SARS-CoV-2. Eur Rev Med Pharmacol Sci. 2020; 24(13):7494-7496. doi: 10.26355/eurrev202007-21921.

[18] Jensen SA, Sorensen JB. 5-fluorouracil-based therapy induces endovascular injury having potential significance to development of clinically overt cardiotoxicity. Cancer Chemother Pharmacol. 2012; 69(1):57-64.

[19] J.L. Fernandes, R. de Oliveira, R.L. Mamoni, O.R. Coelho, J.C. Nicolau, M. Blotta, Jr Serrano CV. Pentoxifylline reduces pro-inflammatory and increases anti-inflammatory activity in patients with coronary artery disease-a randomized placebo-controlled study. Atherosclerosis, 2008; 196(1):434-442.

[20] Polk A, Vaage-Nilsen M, Vistisen K, Nielsen DL. Cardiotoxicity in cancer patients treated with 5-fluorouracil or capecitabine: a systematic review of incidence, manifestations and predisposing factors. Cancer Treat Rev. 2013; 39(8):974-84. doi: 10.1016/j.ctrv.2013.03.005

[21] Wall GC, Smith HL, Trump MW, Mohr JD, DuMontier SP, Sabates BL, Ganapathiraju I, Kable TJ. Pentoxifylline or theophylline use in hospitalized COVID-19 patients requiring oxygen support. Clin Respir J. 2021; 15(7):843-846. doi: 10.1111/crj.13363. 